Pacific Journal of Mathematic 


\title{
OUTER GALOIS THEORY FOR SEPARABLE ALGEBRAS
}

\author{
H. F. KREIMER
}

Let $G$ be a finite group of automorphisms of a ring $A$ which has identity element. Let $C$ be the center of $A$, let $\Gamma$ be the subring of $G$-invariant elements of $A$, and assume that $C$ is a separable extension of $C \cap \Gamma$. In the first section of this paper, it is shown that every finite group of automorphisms of $A$ over $\Gamma$ is faithfully represented as a group of automorphisms of $C$ by restriction if, and only if, $A=C \otimes_{C{ }_{\Gamma} \Gamma} \Gamma$. Moreover, suppose that $A=C \otimes_{C \cap \Gamma} \Gamma$ and $\Omega$ is a subring of $\Lambda$ such that $\Gamma \subseteq \Omega \subseteq \Lambda$. Then there exists a finite group $H$ of automorphisms of $A$ such that $\Omega$ is the subring of $H$ invariant elements of $A$ if, and only if, $C \cap \Omega$ is a separable extension of $C \cap \Gamma$ and $\Omega=(C \cap \Omega) \otimes_{c \cap \Gamma} \Gamma$.

Let $R$ be a commutative ring with identity element; and assume now that $A$ is a separable algebra over $R$ and $G$ is a finite group of automorphisms of the $R$-algebra $\Lambda$. In the second section of this paper, it is shown that $C$ is the centralizer of $\Gamma$ in $\Lambda$ if, and only if, $A=C \otimes_{C \cap \Gamma} \Gamma$. Moreover, suppose that $A=C \otimes_{C \cap \Gamma} \Gamma$ and $\Omega$ is a subalgebra of $A$ such that $\Gamma \leqq \Omega \cong \Lambda$. Then there exists a finite group $H$ of automorphisms of $A$ such that $\Omega$ is the subalgebra of $H$-invariant elements of $A$ if, and only if, $\Omega$ is a separable algebra over $R$.

These results are obtained without the assumption of no nontrivial idempotent elements of $C$, which is required for the KanzakiDeMeyer Galois theory of separable algebras. Moreover, these results extend the Villamayor-Zelinsky Galois theory of commutative rings in the same way that the results of Kanzaki and DeMeyer extend the Chase-Harrison-Rosenberg Galois theory of commutative rings.

1. Galois theory. Throughout this paper, ring will mean ring with identity element and subring of a ring will mean subring which contains the identity element of the ring. Let $\Gamma$ be a subring of a ring $A$. Call $\Lambda$ a projective Frobenius extension of $\Gamma$ if $\Lambda$ is a finitely generated, projective right $\Gamma$-module and there is a $(\Gamma, \Lambda)$ bimodule isomorphism of $\Lambda$ onto $\operatorname{Hom}_{\Gamma}(\Lambda, \Gamma)$. Call $\Lambda$ a separable extension of $\Gamma$ if the $(\Lambda, \Lambda)$-bimodule epimorphism of $\Lambda \otimes_{r} \Lambda$ onto $\Lambda$, which is determined by the ring multiplication in $A$, splits. Equivalently, $A$ is a separable extension of $\Gamma$ if there exist a positive integer $n$ and elements $x_{i}, y_{i}$ of $\Lambda$, for $1 \leqq i \leqq n$, such that $\sum_{i=1}^{n} x_{i} y_{i}=1$ and $\sum_{i=1}^{n} a x_{i} \otimes y_{i}=\sum_{i=1}^{n} x_{i} \otimes y_{i} a$ in $\Lambda \otimes{ }_{\Gamma} \Lambda$ for every $a \in \Lambda$. Also, let $M$ be a left $\Lambda$-module and let $N$ be a $\Gamma$-submodule of $M$. A canonical $\Lambda$-module homomorphism $\phi$ of $\Lambda \otimes_{\Gamma} N$ into $M$ is determined by the 
correspondence of $a \cdot x$ to $a \otimes x$ for $a \in \Lambda$ and $x \in N$. It will be convenient of write $M=\Lambda \otimes{ }_{r} N$ when $\phi$ is an isomorphism.

Let $G$ be a finite group of automorphisms of a ring 1 , and let $\Gamma$ be the subring of $G$-invariant elements of $\Lambda$. Call $\Lambda$ a Galois extension of $\Gamma$ relative to $G$ if there exist a positive integer $n$ and elements $x_{i}, y_{i}$ of $\Lambda, 1 \leqq i \leqq n$, such that $\sum_{i=1}^{n} x_{i} \cdot \sigma\left(y_{i}\right)=\delta_{1, \sigma}$ for all $\sigma \in G$. If $\Lambda$ is a Galois extension of $\Gamma$ relative to $G$, then $\Lambda$ is a separable extension of $\Gamma$ by [8, Proposition 1.3]. Let $C$ be the center of $\Lambda$. If $A$ is a Galois extension of $\Gamma$ relative to $G$ and $C$ is the centralizer of $\Gamma$ in $\Lambda$, call $\Lambda$ an outer Galois extension of $\Gamma$ relative to $G$. A generalization of the concept of outer Galois extension is that of outer semi-Galois extension given in [7, Definition 2.4]. $\Lambda$ will be called an outer semi-Galois extension of $\Gamma$ if, in addition to the assumptions stated at the beginning of this paragraph, $\Lambda$ is a separable extension of $\Gamma$ and $C$ is the centralizer of $\Gamma$ in $\Lambda$. Finally, we note that, if $S$ is a $G$-stable subring of $\Lambda$; then a homomorphism of $G$ onto a finite group $\bar{G}$ of automorphisms of $S$ is obtained by restricting each element of $G$ to $S$, and $S \cap \Gamma$ is the subring of $\bar{G}$-invariant elements of $S$.

For the remainder of the paper, let $G$ be a finite group of automorphisms of a ring $\Lambda$, let $\Gamma$ be the subring of $G$-invariant elements of $\Lambda$, and let $C$ be the center of $\Lambda$.

THeOREM 1.1. If $S$ is a G-stable subring of $C$ such that $S$ is a separable extension of $S \cap \Gamma$, then the following statements are equivalent.

(i) $\mathrm{C}=S \otimes_{S \cap \Gamma}(C \cap \Gamma)$ and $\Lambda=C \otimes_{c \cap \Gamma} \Gamma$.

(ii) $\Lambda=S \otimes_{s \cap \Gamma} \Gamma$.

(iii) An isomorphism of the group of all automorphisms of $A$ over $\Gamma$ for which $S$ is stable onto the group of all automorphisms of $S$ over $S \cap \Gamma$ is obtained by restricting each automorphism of $\Lambda$ to $S$.

(iv) Every finite group of automorphisms of $\Lambda$ over $\Gamma$ for which $S$ is stable is faithfully represented as a group of automorphisms of $S$ by restriction.

Proof. It is evident that statement (i) implies statement (ii). If $\Lambda=S \otimes_{S \cap \Gamma} \Gamma$, then every automorphism of $S$ over $S \cap \Gamma$ may be extended to an automorphism of $\Lambda$ over $\Gamma$ and the identity map on $\Lambda$ is the only automorphism of $\Lambda$ over $\Gamma$ which restricts to the identity map on $S$. With these observations it is easily verified that statement (ii) implies statement (iii). Clearly statement (iii) implies statement (iv), and it only remains to verify that statement (iv) implies statement (i). Since $S$ is a commutative ring and a separable 
extension of $S \cap \Gamma, S$ is an outer semi-Galois extension of $S \cap \Gamma$. Let $e$ be an idempotent element of $S$ and $\sigma$ be an element of $G$ such that $\sigma(e \cdot a)=e \cdot a$ for all $a \in S$. An automorphism $\bar{\sigma}$ of $\Lambda$ over $\Gamma$ is defined by the rule $\bar{\sigma}(a)=\sigma(e \cdot a)+(1-e) \cdot a$ for $a \in \Lambda$. Let $H$ be the group of automorphisms of $\Lambda$ over $\Gamma$ which is generated by $\bar{\sigma}$. Since $G$ is a finite group, $\sigma$ has finite order. Therefore $\bar{\sigma}$ has finite order and $H$ is a finite group. Moreover, each element of $S$ is $H$-invariant; and $H$ is faithfully represented as a group of automorphisms of $S$ by restriction, only if $\bar{\sigma}(a)=a$ and, hence, $\sigma(e \cdot a)=e \cdot a$ for all $a \in \Lambda$. Since $C$ is stable for any group of automorphisms of $A$, the following lemma may be applied with $T=C$ to establish that statement (iv) implies statement (i).

Lemma 1.2. Let $S, T$ be G-stable subrings of $A$ such that $S \cong T$ and $S$ is an outer semi-Galois extension of $S \cap \Gamma$. Assume that whenever $e$ is a central idempotent of $S$ and $\sigma$ is an element of $G$ such that $\sigma(e \cdot a)=e \cdot a$ for all $a \in S$, then $\sigma(e \cdot a)=e \cdot a$ for all $a \in \Lambda$. Then $T=S \otimes_{S_{\cap \cap \Gamma}}(T \cap \Gamma)$ and $\Lambda=T \otimes_{T \cap \Gamma} \Gamma$.

Proof. By hypothesis, $S$ is a separable extension of $S \cap \Gamma$. Let $n$ be a positive integer and let $x_{i}, y_{i}$ be elements of $S$ for $1 \leqq i \leqq n$, such that $\sum_{i=1}^{n} x_{i} y_{i}=1$ and $\sum_{i=1}^{n} a x_{i} \otimes y_{i}=\sum_{i=1}^{n} x_{i} \otimes y_{i} a$ in $S \otimes{ }_{S \cap \Gamma} S$ for every $a \in S$. Setting $e_{\sigma}=\sum_{i=1}^{n} x_{i} \cdot \sigma\left(y_{i}\right), a \cdot e_{\sigma}=e_{\sigma} \cdot \sigma(a)$ for $a \in S$ and $\sigma \in G$. Therefore $e_{\sigma}$ is an element of the centralizer of $S \cap \Gamma$ in $S$, which is the center of $S$, for $\sigma \in G$. Moreover

$$
e_{\sigma}^{2}=\sum_{i=1}^{n} e_{\sigma} \cdot x_{i} \cdot \sigma\left(y_{i}\right)=\sum_{i=1}^{n} x_{i} \cdot e_{\sigma} \cdot \sigma\left(y_{i}\right)=\sum_{i=1}^{n} x_{i} \cdot y_{i} \cdot e_{\sigma}=e_{\sigma}
$$

for $\sigma \in G$. Thus $\left\{\sigma\left(e_{\tau}\right) \mid \sigma, \tau \in G\right\}$ is a finite set of central idempotents in $S$, and it generates a finite, $G$-stable subalgebra $E$ of the Boolean algebra of all central idempotents in $S$. Letting $M$ be the set of minimal elements in $E$; $M$ is a finite, $G$-stable set of pairwise orthogonal idempotents such that $\sum_{e \in M} e=1$. For $e \in M$ and $\sigma \in G$, let e $\sigma$ denote the mapping $a m e \cdot \sigma(a), a \in \Lambda$; and let

$$
N=\{e \sigma \mid e \in M, \sigma \in G\} .
$$

The mapping $\alpha \leadsto \sigma \circ \alpha, \alpha \in N$, is a permutation on $N$ for each $\sigma \in G$. Consequently, letting $\gamma$ be the sum of the distinct elements of $N ; \gamma$ is a left $S \cap \Gamma$-module endomorphism of $\Lambda$, the image of which must be contained in $\Gamma$. Since $S$ and $T$ are $G$-stable, $\gamma$ must map $S$ into $S \cap \Gamma$ and $T$ into $T \cap \Gamma$. If, for $e \in M$ and $\sigma \in G$,

$$
\sum_{i=1}^{n} x_{i} \cdot e \cdot \sigma\left(y_{i}\right)=e \cdot e_{\sigma}=e_{\sigma} \cdot \sigma(e)
$$


is not zero; then $e=e \cdot e_{\sigma}=e_{\sigma} \cdot \sigma(e)=\sigma(e)$ since $e$ and $\sigma(e)$ are minimal elements of $E, \sigma(e \cdot a)=e_{\sigma} \cdot \sigma(e \cdot a)=e \cdot a \cdot e_{\sigma}=e \cdot a$ for all $a \in S$, and by hypothesis $\sigma(e \cdot a)=e \cdot a$ for all $a \in \Lambda$. Therefore $\sum_{i=1}^{n} x_{i} \cdot \gamma\left(y_{i} a\right)=$ $\sum_{e \in M} e \cdot a=a$ for all $a \in \Lambda$. It is now readily verified that the canonical left $S$-module homomorphism of $S \otimes_{S \cap \Gamma} \Gamma$ into $\Lambda$ has an inverse which is the mapping $a m \sum_{i=1}^{n} x_{i} \otimes \gamma\left(y_{i} a\right), a \in \Lambda$; and the canonical left $S$ module homomorphism of $S \otimes_{S \cap \Gamma}(T \cap \Gamma)$ into $T$ has an inverse which is the mapping $a m \sum_{i=1}^{n} x_{i} \otimes \gamma\left(y_{i} a\right), a \in T$. Thus $\Lambda=S \otimes_{S \cap r} \Gamma$ and $T=S \otimes_{S \cap \Gamma}(T \cap \Gamma)$. Since $S \otimes_{S \cap \Gamma} \Gamma$ is naturally isomorphic to $S \otimes_{S \cap \Gamma}(T \cap \Gamma) \otimes_{T \cap \Gamma} \Gamma, \Lambda=T \otimes_{T \cap \Gamma} \Gamma$.

If $S$ has no central idempotents other than 0 and 1 , then the hypotheses of Lemma 1.2 are equivalent to the requirements that $S$ and $T$ be $G$-stable subrings of $\Lambda$ such that $S \subseteq T, S$ be an outer semi-Galois extension of $S \cap \Gamma$, and $G$ be faithfully represented as a group of automorphisms of $S$ by restriction. The following example, however, shows that in general the conclusion of Lemma 1.2 cannot be obtained if only these latter conditions are assumed.

EXAmple 1.3. Let $\Lambda$ be the ring of all complex $3 \times 3$ matrices of the form $\left(\begin{array}{lll}a & b & 0 \\ c & d & 0 \\ 0 & 0 & g\end{array}\right)$, and set $\sigma\left(\begin{array}{lll}a & b & 0 \\ c & d & 0 \\ 0 & 0 & g\end{array}\right)=\left(\begin{array}{rrr}a & -b & 0 \\ -c & d & 0 \\ 0 & 0 & \bar{g}\end{array}\right)$ and $\tau\left(\begin{array}{lll}a & b & 0 \\ c & d & 0 \\ 0 & 0 & g\end{array}\right)=$ $\left(\begin{array}{ccc}\bar{a} & \bar{b} & 0 \\ \bar{c} & \bar{d} & 0 \\ 0 & 0 & \bar{g}\end{array}\right) . \sigma$ and $\tau$ are automorphisms of $\Lambda$, and they generate a subgroup $G$ of order four in the group of all automorphisms of $\Lambda$. The subring $\Gamma$ of $G$-invariant elements of $\Lambda$ consists of all real, diagonal $3 \times 3$ matrices, and the center $C$ of $\Lambda$ consists of all complex, diagonal $3 \times 3$ matrices of the form diag $\{a, a, b\}$. Take $S=T=C . C$ is a commutative $G$-stable subring of $A$ and $G$ is faithfully represented as a group of automorphisms of $C$ by restriction. Moreover it may be verified that $C$ is a Galois extension of $C \cap \Gamma$ with respect to the group $H$ of automorphisms of $C$ generated by the restriction of $\tau$ to $C$, but $A \neq C \otimes_{c_{n i} \Gamma} \Gamma$. In fact, setting $\phi\left(\begin{array}{lll}a & b & 0 \\ c & d & 0 \\ 0 & 0 & g\end{array}\right)=\left(\begin{array}{rrr}a & -b & 0 \\ -c & d & 0 \\ 0 & 0 & g\end{array}\right), \phi$ is a nontrivial automorphism of $\Lambda$ over $\Gamma$ which restricts to the identity map on $C$.

The remaining results of this section are directed toward developing a Galois theory for a ring $\Lambda$ which satisfies any of the four equivalent statements of Theorem 1.1. 
Lemma 1.4. If $S$ is a G-stable subring of $C$ such that $S$ is a separable extension of $S \cap \Gamma$ and $\Lambda=S \bigotimes_{{ }_{S \cap} \Gamma} \Gamma$, then $\Lambda$ is an outer semi-Galois extension of $\Gamma$ and $\Gamma$ is a $(\Gamma, \Gamma)$-bimodule direct summand of $\Lambda$.

Proof. Let $S$ be a $G$-stable subring of $C$ such that $S$ is a separable extension of $S \cap \Gamma$ and $\Lambda=S \otimes_{S \cap \Gamma} \Gamma$. Then one may readily verify that $\Lambda$ is a separable extension of $\Gamma$ and the centralizer of $\Gamma$ in $\Lambda$ is $C$. Therefore $\Lambda$ is an outer semi-Galois extension of $\Gamma$. Furthermore, since $S$ is a commutative ring, $S$ is an outer semiGalois extension of $S \cap \Gamma$; and, by [7, Th. 3.2], $S$ is a projective Frobenius extension of $S \cap \Gamma$. In particular, $S$ is a finitely generated, projective module over $S \cap \Gamma$; and it follows from [1, Proposition A. 3] and [9, Proposition 1] that $S \cap \Gamma$ is an $S \cap \Gamma$-module direct summand of $S$. Therefore $\Gamma$ is a $(\Gamma, \Gamma)$-bimodule direct summand of $\Lambda=S \otimes_{S \cap \Gamma} \Gamma$.

LEMma 1.5. Let $\bar{G}$ be a finite group of automorphisms of a commutative ring $S$, let $R$ be the subring of $\bar{G}$-invariant elements of $S$, and assume that $S$ is a separable extension of $R$. For an intermediate ring $T, R \cong T \subseteq S$, the following statements are equivalent.

(i) There exists a finite group $H$ of automorphisms of $S$ such that $T$ is the subring of $H$-invariant elements of $S$.

(ii) $S$ is a projective Frobenius extension of $T$.

(iii) $T$ is a separable extension of $R$.

Proof. Apply Lemma 1.4 with $\Lambda=S=C$ and $\Gamma=R$ to establish that $S$ is an outer semi-Galois extension of $R$ and $R$ is an $R$-module direct summand of $S$. The equivalence of statements (i) and (ii) follows from [7, Th. 3.3]. But it is a consequence of [7, Th. 2.3] and [10,3.15] that $S$ is a weakly Galois $R$-algebra, and the equivalence of statements (i) and (iii) follows from [10, Th. 3.8].

THeOREM 1.6. Let $S$ be a G-stable subring of $C$ such that $S$ is a separable extension of $S \cap \Gamma$ and $\Lambda=S \otimes_{S \cap \Gamma} \Gamma$; and let $\Omega$ be a subring of $\Lambda$ such that $\Gamma \leqq \Omega \subseteq \Lambda$. There exists a finite group $H$ of automorphisms of $A$ such that $S$ is $H$-stable and $\Omega$ is the subring of $H$-invariant elements in $\Lambda$ if, and only if, $S \cap \Omega$ is a separable extension of $S \cap \Gamma$ and $\Omega=(S \cap \Omega) \otimes_{s \cap \Gamma} \Gamma$.

Proof. Suppose $H$ is a finite group of automorphisms of $\Lambda$ such that $S$ is $H$-stable and $\Omega$ is the subring of $H$-invariant elements in 1. Then $S \cap \Omega$ is the subring of $H$-invariant elements in $S$, and 
$S \cap \Omega$ is a separable extension of $S \cap \Gamma$ by Lemma 1.5. Also by Lemma 1.5, $S$ is a finitely generated, projective module over $S \cap \Omega$; and it follows from [1, Proposition A. 3] and [7, Lemma 1.6] that $S$ is a faithfully flat module over $S \cap \Omega$. Since $\Lambda=S \otimes_{S \cap \Gamma} \Gamma$ and $\Gamma \subset \Omega$, every group of automorphisms of $\Lambda$ over $\Omega$ for which $S$ is stable is faithfully represented as a group of automorphisms of $S$ by restriction. Therefore $\Lambda=S \otimes_{S \cap \Omega} \Omega$ by Theorem 1.1. But $\Lambda=S \bigotimes_{S \cap \Omega}(S \cap \Omega) \bigotimes_{S \cap \Gamma} \Gamma$ also. Since $\mathrm{S}$ is a faithfully flat module over $S \cap \Omega, \Omega=(S \cap \Omega) \otimes_{S \cap \Gamma} \Gamma$.

Conversely, suppose $S \cap \Omega$ is a separable extension of $S \cap \Gamma$ and $\Omega=(S \cap \Omega) \otimes_{S \cap \Gamma} \Gamma$. By Lemma 1.5, there exists a finite group $H$ of automorphisms of $S$ such that $S \cap \Omega$ is the subring of $H$-invariant elements in $S$. Since $\Lambda=S \otimes_{S_{\cap} \Gamma} \Gamma$, there is a unique extension of $H$ to a group of automorphisms of $\Lambda$ over $\Gamma$. Let $\Omega^{\prime}$ be the subring of $H$-invariant elements in $\Lambda . \Gamma \subseteq \Omega^{\prime}$; and, by the first part of this proof, $\Omega^{\prime}=\left(S \cap \Omega^{\prime}\right) \otimes_{S \cap \Gamma} \Gamma$. But $S \cap \Omega^{\prime}$ is the subring of $H$-invariant elements in $S$, so $S \cap \Omega^{\prime}=S \cap \Omega$ and

$$
\Omega^{\prime}=\left(S \cap \Omega^{\prime}\right) \otimes_{S \cap \Gamma} \Gamma=(S \cap \Omega) \otimes_{S \cap \Gamma} \Gamma=\Omega .
$$

If $S$ is a $G$-stable subring of $C$ such that $S$ is a separable extension of $S \cap \Gamma$ and $\Lambda=S \otimes_{S \cap \Gamma} \Gamma$; then $C=S \otimes_{s \cap \Gamma}(C \cap \Gamma)$ and $\Lambda=$ $C \otimes_{C \cap \Gamma} \Gamma$ by Theorem 1.1, and $C$ is a separable extension of $C \cap \Gamma$ by [2, Corollary 1.6]. Since $C$ is stable for any group of automorphisms of $\Lambda, S$ may be replaced by $C$ in the preceding considerations. The following corollary is stated for comparison with Lemma 1.5 .

Corollary 1.7. Assume that $C$ is a separable extension of $C \cap \Gamma$ and every finite group of automorphisms of $\Lambda$ over $\Gamma$ is faithfully represented as a group of automorphisms of $C$ by restriction. For a subring $\Omega$ of $\Lambda$ such that $\Gamma \leqq \Omega \subseteq \Lambda$, the following statements are equivalent.

(i) There exists a finite group $H$ of automorphisms of $\Lambda$ such that $\Omega$ is the subring of $H$-invariant elements of $\Lambda$.

(ii) $\Lambda$ is a projective Frobenius extension of $\Omega$.

(iii) $C \cap \Omega$ is a separable extension of $C \cap \Gamma$ and

$$
\Omega=(C \cap \Omega) \otimes_{{ }_{C \cap} \Gamma} \Gamma \text {. }
$$

Proof. Since every finite group of automorphisms of $\Lambda$ over $\Gamma$ is faithfully represented as a group of automorphisms of $C$ by restriction, $A=C \otimes_{C \cap \Gamma} \Gamma$ by Theorem 1.1. Therefore $\Lambda$ is an outer semiGalois extension of $\Gamma$ and $\Gamma$ is a $(\Gamma, \Gamma)$-bimodule direct summand of $\Lambda$ by Lemma 1.4. Statements (i) and (ii) are equivalent by [7, Th. 3.3], and statements (i) and (iii) are equivalent by Theorem 1.6. 
2. Separable algebras. In this section, let $\Lambda$ be an algebra over a commutative ring $R$ and let $G$ be a finite group of automorphisms of the $R$-algebra $\Lambda$. Let $\Gamma$ be the subalgebra of $G$-invariant elements of $\Lambda$ and let $C$ be the center of $\Lambda$. The results of the preceding section may be sharpened if $A$ is a separable algebra over $R$. Thus let $A$ be a separable algebra over $R$. Then $A$ is a separable extension of $C$ and $C$ is a separable algebra over $R$ by [2, Th. 2.3]. Clearly $R \cdot 1 \subseteq C \cap \Gamma \subseteq \Gamma$; and, consequently, $\Lambda$ is a separable extension of $\Gamma$ and $C$ is a separable extension of $C \cap \Gamma$.

Proposition 2.1. If $\Lambda$ is a separable extension of $C$ and $C$ is the centralizer of $\Gamma$ in $\Lambda$, then the group of all automorphisms of $\Lambda$ over $\Gamma$ is faithfully represented as a group of automorphisms of $C$ by restriction.

Proof. Assume that $A$ is a separable extension of $C$ and $C$ is the centralizer of $\Gamma$ in $\Lambda$; but suppose that the group of all automorphisms of $\Lambda$ over $\Gamma$ is not faithfully represented as a group of automorphisms of $C$ by restriction, and let $\eta$ be a nontrivial automorphism of $\Lambda$ over $\Gamma$ which restricts to the identity map on $C$. Let $a$ be an element of $\Lambda$ such that $\eta(\alpha) \neq a$, let $m$ be a maximal ideal of $C$ which contains the set $\{x \in C \mid x \cdot(\eta(a)-a)=0\}$, and let $C_{m}$ be the quotient ring of $C$ with respect to the multiplicative system $C-m . \quad \Lambda \otimes{ }_{c} C_{m}$ is a central separable algebra over $C \otimes{ }_{c} C_{m}=C_{m}$ by [2, Corollary 1.6], and $\eta \otimes 1$ is an automorphism of $\Lambda \otimes{ }_{c} C_{m}$ over $C_{m}$. Since $C_{m}$ is a local ring, $\eta \otimes 1$ is an inner automorphism by [2, Th. 3.6 and the remark which follows it]. Let $w \otimes 1 / s, w \in \Lambda$ and $s \in C-m$, be a unit in $A \otimes{ }_{C} C_{m}$ such that $w \cdot \eta(x) \otimes 1 / s=x w \otimes 1 / \mathrm{s}$ for all $x \in \Lambda$. $A$ is a finitely generated module over $C$ by [2, Th. 2.1]; so let $n$ be a positive integer and $\left\{b_{i} \in \Lambda \mid 1 \leqq i \leqq n\right\}$ be a set of generators for the $C$-module $\Lambda$. Since $w \cdot \eta\left(b_{i}\right) \otimes 1 / s=b_{i} \cdot w \otimes 1 / s$; there exists $t_{i} \in C-m$ such that $t_{i} \cdot\left(w \cdot\left(b_{i}\right)-b_{i} \cdot w\right)=0,1 \leqq i \leqq n$, by [3, $\S 2$, No. 2, Proposition 4]. Letting $t=\prod_{i=1}^{n} t_{i}$, it is easily verified that $t \in C-m$ and $t w \cdot \eta(x)=x t w$ for all $x \in \Lambda$. Therefore $t w$ is an element of the centralizer of $\Gamma$ in $\Lambda$, which is $C ; w \otimes 1 / s=$ $t w \otimes 1 /(t s)$ is a unit in the center of $\Lambda \otimes{ }_{c} C_{m}$; and, consequently, $\eta(x) \otimes 1=x \otimes 1$ for all $x \in \Lambda$. In particular $\eta(a) \otimes 1=a \otimes 1$; and, by [3, $\S 2$, No. 2, Proposition 4], there exists $u \in C-m$ such that $u \cdot(\eta(a)-a)=0$. But such an element $u$ cannot exist by the choice of $m$, and the proposition follow from this contradiction.

COROLlaRY 2.2. If $A$ is a separable algebra over $R$, then the following statements are equivalent.

(i) $C$ is the centralizer of $\Gamma$ in $\Lambda$. 
(ii) $\Lambda$ is an outer semi-Galois extension of $\Gamma$.

(iii) $\Lambda=C \otimes_{\mathrm{C} \cap \Gamma} \Gamma$.

Proof. Assume that $\Lambda$ is a separable algebra over $R$. Then $\Lambda$ is a separable extension of $\Gamma$, and therefore statements (i) and (ii) are equivalent. Moreover, $A$ is a separable extension of $C$ and $C$ is a separable extension of $C \cap \Gamma$. It follows from Proposition 2.1 and Theorem 1.1 that statement (i) implies statement (iii). Clearly statement (iii) implies statement (i).

THEOREM 2.3. Let $A$ be a separable algebra over $R$ such that $\Lambda=C \otimes_{{ }_{0} \Gamma \Gamma} \Gamma$; and let $\Omega$ be a subalgebra of $\Lambda$ such that $\Gamma \subseteq \Omega \subseteq \Lambda$. There exists a finite group $H$ of automorphisms of $\Lambda$ such that $\Omega$ is the subalgebra of $H$-invariant elements of $\Lambda$ if, and only if, $\Omega$ is a separable algebra over $R$.

Proof. $\Lambda$ is an outer semi-Galois extension of $\Gamma$ and $\Gamma$ is a $(\Gamma, \Gamma)$-bimodule direct summand of $\Lambda$ by Lemma 1.4. Since $\Lambda$ is a separable algebra over $R, \Lambda$ is a $(\Lambda, \Lambda)$-bimodule direct summand of $\Lambda \otimes_{R} \Lambda$; and thus $\Gamma$ is a $(\Gamma, \Gamma)$-bimodule direct summand of $\Lambda \otimes_{R} \Lambda$. As a $(\Gamma, \Gamma)$-bimodule, $\Lambda \otimes_{R} \Lambda$ is a left module over the enveloping algebra $\Gamma^{e}=\Gamma \otimes_{R} \Gamma^{0}$ of $\Gamma$; and for any left $\Gamma^{e}$-module $X$ there is a natural isomorphism of $\operatorname{Hom}_{\Gamma e}\left(\Lambda \otimes_{R} \Lambda, X\right)$ onto $\operatorname{Hom}\left({ }_{r} \Lambda, \operatorname{Hom}\left(\Lambda_{\Gamma}, X\right)\right)$. But $\Lambda$ is a projective Frobenius extension of $\Gamma$ by [7, Th. 3.2]; and, therefore, $\Lambda$ is projective as either a left or right $\Gamma$-module. Consequently, $\Lambda \otimes{ }_{R} \Lambda$ must be a projective left $\Gamma^{e}$-module. Therefore $\Gamma$ is a projective left $\Gamma^{e}$-module, and it follows that $\Gamma$ is a separable algebra over $R$.

By Theorem 1.6, there exists a finite group $H$ of automorphisms of $\Lambda$ such that $\Omega$ is the subring of $H$-invariant elements of $\Lambda$ if, and only if, $C \cap \Omega$ is a separable extension of $C \cap \Gamma$ and $\Omega=(C \cap \Omega) \otimes_{{ }_{C}{ }_{\Gamma}} \Gamma$. But if $C \cap \Omega$ is a separable extension of $C \cap \Gamma$ and $\Omega=(C \cap \Omega) \otimes{ }_{C \cap I} \Gamma$, then one may readily verify that $\Omega$ is a separable algebra over $R$. Conversely, suppose $\Omega$ is a separable algebra over $R$. Since $R \cdot 1 \subseteq C \cap \Gamma, \Gamma$ and $\Omega$ are separable extensions of $C \cap \Gamma$; and, since $C$ is the centralizer of $\Gamma$ in $\Lambda, C \cap \Gamma$ is the center of $\Gamma$ while $C \cap \Omega$ is both the centralizer of $\Gamma$ in $\Omega$ and the center of $\Omega$. But then $C \cap \Omega$ is a separable extension of $C \cap \Gamma$ by [2, Th. 2.3], and $\Omega=$ $(C \cap \Omega) \otimes_{C \cap \Gamma} \Gamma$ by [2, Th. 3.1].

\section{BIBLIOGRAPHY}

1. M. Auslander and O. Goldman, Maximal orders, Trans. Amer. Math. Soc. 97 (1960), 1-24. 
2. The Brauer group of a commutative ring, Trans. Amer. Math. Soc. 97 (1960), 367-409.

3. Bourbaki, Algèbre commutative, Chap. 2, Hermann, Paris, 1961.

4. S. U. Chase, D. K. Harrison, and A. Rosenberg, Galois theory and cohomology of commutative rings, Mem. Amer. Math. Soc. 52 (1965), 15-33.

5. F. R. DeMeyer, Some notes on the general Galois theory of rings, Osaka J. Math. 2 (1965), 117-127.

6. T. Kanzaki, On commutor rings and Galois theory of separable algebras, Osaka J. Math. 1 (1964), 103-115.

7. H. F. Kreimer, $A$ note on the outer Galois theory of rings (to appear in Pacific J. Math.)

8. Y. Miyashita, Finite outer Galois theory of non-commutative rings, J. Fac. Sci. Hokkaido Univ. (I) 19 (1966), 114-134.

9. T. Nakayama, On a generalized notion of Galois extension of a ring, Osaka J. Math. 15 (1963), 11-23.

10. O. E. Villamayor and D. Zelinsky, Galois theory with infinitely many idempotents, Nagoya Math. J. 35 (1969), 83-98.

Received February 18, 1969. The author gratefully acknowledges receiving partial support in his research from the Florida State University Research Council and the National Science Foundation under grant GP-8424.

Florida State University 



\section{PACIFIC JOURNAL OF MATHEMATICS}

\section{EDITORS}

\author{
H. SAMELSON \\ Stanford University \\ Stanford, California 94305 \\ Richard Pierce \\ University of Washington \\ Seattle, Washington 98105
}

J. DUGUNDJI

Department of Mathematics

University of Southern California

Los Angeles, California 90007

BASIL GORDON*

University of California

Los Angeles, California 90024

\section{ASSOCIATE EDITORS}
E. F. BECKENBACH
B. H. NeUmanN
F. WOLF
K. YoSHIDA

\section{SUPPORTING INSTITUTIONS}

UNIVERSITY OF BRITISH COLUMBIA CALIFORNIA INSTITUTE OF TECHNOLOGY

UNIVERSITY OF CALIFORNIA MONTANA STATE UNIVERSITY

UNIVERSITY OF NEVADA

NEW MEXICO STATE UNIVERSITY

OREGON STATE UNIVERSITY

UNIVERSITY OF OREGON

OSAKA UNIVERSITY

UNIVERSITY OF SOUTHERN CALIFORNIA
STANFORD UNIVERSITY

UNIVERSITY OF TOKYO

UNIVERSITY OF UTAH

WASHINGTON STATE UNIVERSITY

UNIVERSITY OF WASHINGTON

AMERICAN MATHEMATICAL SOCIETY CHEVRON RESEARCH CORPORATION TRW SYSTEMS

NAVAL WEAPONS CENTER 


\section{Pacific Journal of Mathematics}

\section{Vol. 32, No. $1 \quad$ January, 1970}

Robert Alexander Adams, Compact Sobolev imbeddings for unbounded domains ........................................ 1

Bernhard Amberg, Groups with maximum conditions .................. 9

Tom M. (Mike) Apostol, Möbius functions of order k............... 21

Stefan Bergman, On an initial value problem in the theory of two-dimensional transonic flow patterns ................... 29

Geoffrey David Downs Creede, Concerning semi-stratifiable spaces ...... 47

Edmond Dale Dixon, Matric polynomials which are higher

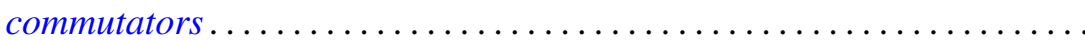

R. L. Duncan, Some continuity properties of the Schnirelmann density.

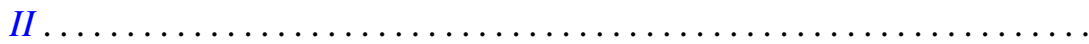

Peter Larkin Duren and Allen Lowell Shields, Coefficient multipliers of $H^{p}$

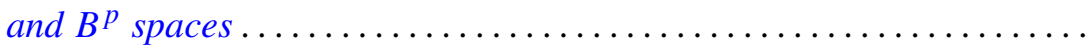

Hector O. Fattorini, On a class of differential equations for vector-valued

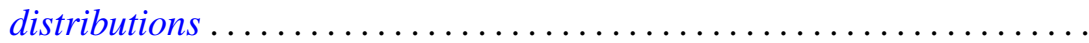

Charles Hallahan, Stability theorems for Lie algebras of derivations. . . . . . 105

Heinz Helfenstein, Local isometries of flat tori ................ 113

Gerald J. Janusz, Some remarks on Clifford's theorem and the Schur

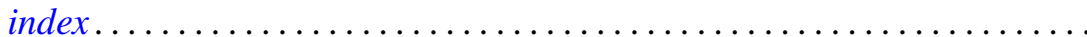

Joe W. Jenkins, Symmetry and nonsymmetry in the group algebras of discrete groups. ...

Herbert Frederick Kreimer, Jr., Outer Galois theory for separable

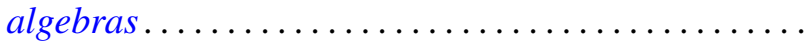

D. G. Larman and P. Mani, On visual hulls

R. Robert Laxton, On groups of linear recurrences. II. Elements of finite order.

Dong Hoon Lee, The adjoint group of Lie groups ...

James B. Lucke, Commutativity in locally compact rings

Charles Harris Scanlon, Rings of functions with certain Lipschitz

$$
\text { properties ............................... }
$$

Binyamin Schwarz, Totally positive differential systems .

James McLean Sloss, The bending of space curves into piecewise helical curves.

James D. Stafney, Analytic interpolation of certain multiplier spaces ...

Patrick Noble Stewart, Semi-simple radical classes.......

Hiroyuki Tachikawa, On left $\mathrm{QF}-3$ rings ...................... 255

Glenn Francis Webb, Product integral representation of time dependent nonlinear evolution equations in Banach spaces.... . . . 